\title{
INTENCIÓN EMPRENDEDORA EN ESTUDIANTES DE PREGRADO DE LA UNIVERSIDAD DE NARIÑO, COLOMBIA ${ }^{1}$
}

\section{ENTREPRENEURIAL INTENTION IN UNDERGRADUATE STUDENTS AT THE UNIVERSITY OF NARIÑO, COLOMBIA \\ INTENÇÃO EMPREENDEDORA EM ESTUDANTES DE GRADUAÇÃO DA UNIVERSIDADE DE NARIÑO, COLOMBIA}

Adriana Aguilera-Castro; Luis Eduardo Benavides-Pupiales; Fabio Jesús Ibarra-Jaramillo

Magíster en Ciencias de la Organización, Universidad del Valle, Colombia. Profesora Titular, Facultad de Ciencias de la Administración, Universidad del Valle, Colombia. Miembro del Grupo de Investigación Humanismo y Gestión. ORCiD: 0000-0001-6483-3712. E-mail: adriana.aguilera@correounivalle.edu.co,Colombia.

Magíster en Administración, Universidad del Valle, Colombia. Docente Tiempo Completo, Programa Administración de Empresas, Universidad CESMAG. ORCiD: 0000-0003-2506-7888. E-mail: lebenavides@ unicesmag.edu.co, Colombia.

Magíster en Administración, Universidad del Valle, Colombia. Docente Hora Cátedra, Programa Administración de Empresas, Universidad de Nariño. ORCiD: 0000-0003-2224-3313 E-mail: fabioij@udenar.edu.co, Colombia.

\section{Recibido: 24 de septiembre de 2020}

DOI: https://doi.org/10.22267/rtend.212202.174

\footnotetext{
${ }^{1}$ Este artículo es producto del proyecto de investigación titulado: "Estudio de las intenciones emprendedoras de los estudiantes de pregrado de la Universidad de Nariño sede Pasto, fundamentado en la Teoría del comportamiento planeado", que fue presentado como trabajo de investigación para optar al título de Magister en Administración de la Universidad del Valle, 2019.
} 


\title{
Resumen
}

La presente investigación aborda un análisis de la intención emprendedora en los estudiantes que están culminando sus estudios de pregrado en la Universidad de Nariño y hacen parte de programas profesionales relacionados con los sectores de clase mundial. El tipo de investigación es exploratoria y descriptiva; se pretende explorar y describir cuales son las creencias de comportamiento, normativas y controladas de los estudiantes y cómo estas creencias influyen en la intención emprendedora. La información se obtuvo de una muestra de 293 estudiantes pertenecientes a diferentes programas de pregrado. Los resultados indican que en la mayoría de los estudiantes que participaron en la investigación, existe una alta intención emprendedora. Su ambiente cercano, especialmente la familia, los impulsa a crear su propia empresa, pero consideran que factores como el poco apoyo estatal, las limitaciones que tiene la universidad para propiciar espacios adecuados para generar las ideas de negocio y el desconocimiento de metodologías de ideación, inciden negativamente en su intención emprendedora.

Palabras clave: empresario; estudiantes universitarios; Colombia; programas educativos. JEL: L26; M1; M10; M13; M19

\begin{abstract}
This research addresses an analysis of the entrepreneurial intention in students who are completing their undergraduate studies at the University of Nariño and are part of professional programs related to world-class sectors. The type of research is exploratory and descriptive; It is intended to explore and describe what are the behavioral, normative and controlled beliefs of students and how these beliefs influence entrepreneurial intention. The information was obtained from a sample of 293 students belonging to different undergraduate programs. The results indicate that in most of the students who participated in the research, there is a high entrepreneurial intention. Their close environment, especially family, encourages them to create their own company, but they consider factors such as little state support, the limitations that the university has to provide adequate spaces to generate business ideas and ignorance of ideation methodologies, negatively affect your entrepreneurial intention.
\end{abstract}

Keywords: businessman; university students; Colombia; educational programs.

JEL: L26; M1; M10; M13; M19 


\section{Resumo}

Esta pesquisa aborda uma análise da intenção empreendedora em alunos que estão concluindo seus estudos de graduação na Universidade de Nariño e fazem parte de programas profissionais relacionados a setores de classe mundial. O tipo de pesquisa é exploratório e descritivo; Pretendese explorar e descrever quais são as crenças comportamentais, normativas e controladas dos alunos e como essas crenças influenciam a intenção empreendedora. As informações foram obtidas junto a uma amostra de 293 alunos pertencentes a diversos programas de graduação. Os resultados indicam que, na maioria dos alunos que participaram da pesquisa, existe uma elevada intenção empreendedora. Seu ambiente próximo, principalmente familiar, os incentiva a criar sua própria empresa, mas consideram fatores como o pouco apoio do Estado, as limitações que a universidade tem para oferecer espaços adequados para gerar ideias de negócios e o desconhecimento das metodologias de ideação, afetam negativamente sua intenção empreendedora.

Palavras-chave: homem de negocios; estudantes universitarios; Colômbia; programas educativos. JEL: L26; M1; M10; M13; M19

\section{Introducción}

El emprendimiento se ha convertido en una de las prioridades estratégicas de los gobiernos y las distintas instituciones que buscan el desarrollo económico y social de las regiones y de los países. Desde esta perspectiva, las universidades como ejes de investigación, centros de educación y espacios privilegiados para el encuentro social, son claves para promover y desarrollar una cultura de emprendimiento. Existen grandes expectativas respecto a los estudiantes universitarios como creadores de empresas de impacto social y con alto valor agregado, como ocurre por ejemplo con las empresas de base tecnológica (Gómez, 2019).

Estas expectativas acerca de los estudiantes universitarios como emprendedores y empresarios del futuro, hacen necesario conocer sus intenciones de emprendimiento; en particular, es fundamental comprender qué los motiva a crear su propia empresa, así como reconocer el papel de la universidad y el entorno social en la formación no solo de las intenciones de emprendimiento, sino también de las capacidades para que las empresas que se generen sean sostenibles en el tiempo. A 
continuación se presentan de manera general, algunos aspectos conceptuales importantes para el desarrollo de este trabajo.

El concepto de emprendimiento se estableció por primera vez en el siglo XVIII y su significado ha evolucionado desde entonces. Algunos autores lo relacionan con comenzar un negocio propio, pero la mayoría de los economistas creen que es más que eso. Para algunos economistas, el empresario está dispuesto a asumir el riesgo de una nueva empresa si existe una posibilidad significativa de obtener ganancias; otros enfatizan el papel del empresario como un innovador que comercializa su innovación; y algunos más afirman que los empresarios desarrollan nuevos bienes o procesos que el mercado demanda y que actualmente no se suministran. (Gutama y Jiménez, 2019; Gómez, 2019; Sánchez et al., 2017; Azqueta, 2017)

No existe una definición universal de emprendimiento; en la literatura se encuentra que el término "Entrepreneurship" se asocia con el espíritu empresarial. El primer autor que define el emprendimiento es Schumpeter en 1934, quien en su Teoría del desenvolvimiento económico, da a entender que el emprendedor es un agente dentro de un proceso de transformaciones continuas en la organización de la producción, que configuran un avance no lineal de la sociedad. Para Thornton (1999) el emprendimiento se incorporó a la sociología con Weber en 1904, describiendo cómo el comportamiento económico específico de los individuos condujo al auge del capitalismo. El "emprendedor" puede ser entendido como aquella persona que pone en marcha una iniciativa empresarial, crea su propia empresa, solo o asociado con otros promotores, asumiendo los riesgos financieros que esto supone, aportando su trabajo y ocupándose de la dirección de la empresa (Moriano et al., 2001).

En cuanto a la intención emprendedora, se puede definir como un proceso necesario antes de llevar a cabo una acción. Las investigaciones que se han llevado a cabo sobre comportamiento afirman que la intención tiene una mayor importancia como explicación del emprendimiento, que otros factores de carácter psicológico (Bird, 1988). La intención emprendedora es una actitud favorable para optar por la opción de creación de una nueva empresa o la creación de valor al interior de organizaciones ya existentes (intraemprendimiento). Por consiguiente, la intención emprendedora es un fenómeno importante de estudio para las universidades. (Valencia et al., 2016; Durán-Aponte 
y Arias-Gómez, 2015; Sánchez et al., 2005). La intención es un revelador importante del comportamiento planeado, puesto que la decisión de hacerse emprendedor puede considerarse voluntaria y consciente (Baggozi y Baumgartner, 1989). Davidsson (1995) sostiene que las intenciones emprendedoras no predicen correctamente la elección de las personas de crear sus propias empresas, debido a que ésta decisión se supone planeada desde hace algún tiempo y estaría precedida por una intención de hacerlo y en ocasiones, difiere del comportamiento real. Por tanto, sería más valiosa la "convicción" de convertir el emprendimiento en opción de vida.

La intención emprendedora se aborda mediante el uso de modelos de intención que plantean un marco conceptual para la comprensión del proceso emprendedor. Dos modelos que se pueden destacar en la literatura de emprendimiento son: el Modelo Social del Evento Emprendedor (SEE) de Shapero y Sokol (1982) y la Teoría de Comportamiento Planeado (TCP) de Ajzen (1991). La razón fundamental para el uso frecuente de estos modelos según Osorio y Londoño (2015), es la capacidad que tienen para superponerse y su compatibilidad.

La acción emprendedora individual es relevante para el emprendimiento y sin ella "simplemente no habría emprendedor ni nuevas empresas" (Baron, 2007, p. 167). La intención de ser emprendedor está influenciada por varios factores, entre ellos, las características de la personalidad, los factores demográficos y las características ambientales. Las características de personalidad, como la autoeficacia y la necesidad de logro, develan un importante interés predictor en el emprendimiento. Los factores demográficos como la edad, el género, los antecedentes educativos y la experiencia laboral que tienen los individuos, también son importantes. Para los estudiantes que pertenecen a las generaciones actuales, su interés en la iniciativa empresarial alienta la aparición de nuevos empresarios potenciales; esto implica una valoración más fuerte por el trabajo, están dispuestos a asumir los riesgos y a encontrar nuevas formas de generar ingresos. (Valencia et al., 2016).

En la Tabla 1 se relacionan algunos enfoques clásicos que intentan explicar la voluntad de emprender en los individuos; se mencionan estos enfoques en particular, porque exponen elementos relacionados con la intención, iniciativa y motivación para emprender. 


\section{Tabla 1}

Teorías Clásicas que explican la decisión de crear empresa

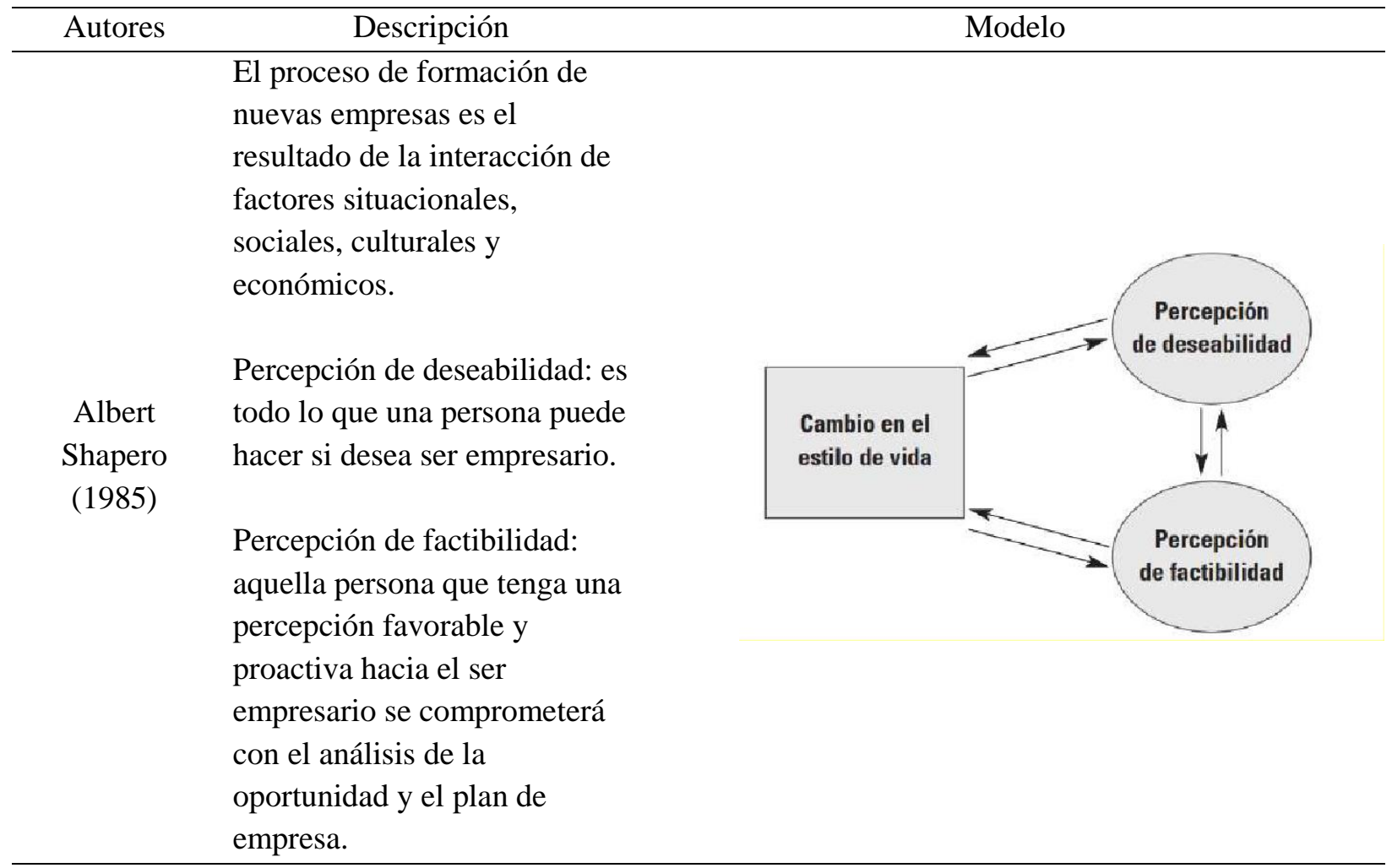

David McClelland (1961)
Esta teoría sostiene que el hombre no realiza actividades económicas únicamente por tener resultados económicos, sino que aparte de esto hay otros componentes como: la necesidad de logro, la necesidad de afiliación y la necesidad de poder/influencia: y que estos factores a su vez son responsables del crecimiento económico.

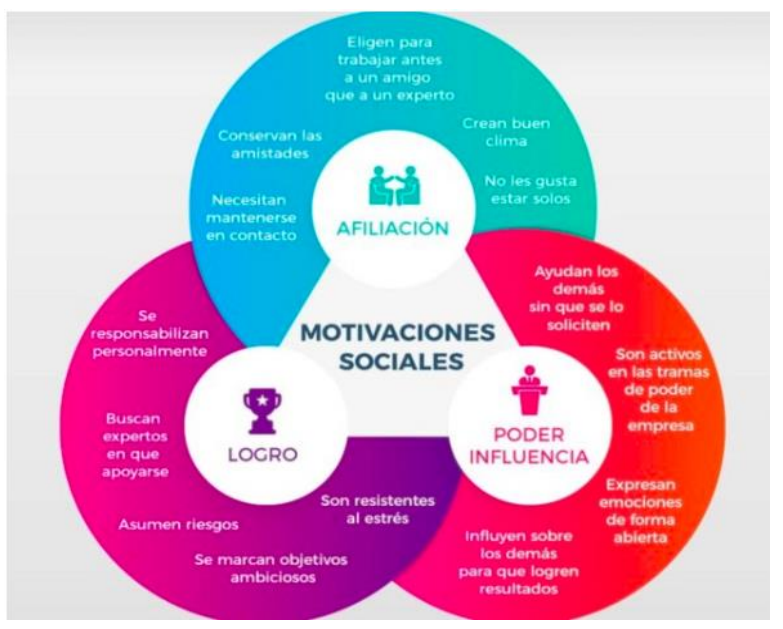


Intención emprendedora en estudiantes de pregrado de la Universidad de Nariño, Colombia Adriana Aguilera-Castro; Luis Eduardo Benavides-Pupiales; Fabio Jesús Ibarra-Jaramillo

\begin{tabular}{cl}
\hline \multicolumn{3}{c}{$\begin{array}{l}\text { Componentes básicos de } \\
\text { iniciativa emprendedora según } \\
\text { Gibb: }\end{array}$} \\
$\begin{array}{cl}\text { Alan Gibb } \\
(1988)\end{array}$ & a) Motivación y \\
& determinación \\
& b) Idea y mercado \\
& c) Recursos \\
d) Habilidades & Motivación y determinación
\end{tabular}

Se basa en la identificación de una serie de etapas que el empresario potencial debe ir pasando antes de llegar a tener su empresa en funcionamiento:

Rodrigo

Varela

Etapa motivacional: Gusto

(2008)

Etapa situacional:

Oportunidad

Etapa de decisión: Decisión

Etapa analítica: Plan de

empresa

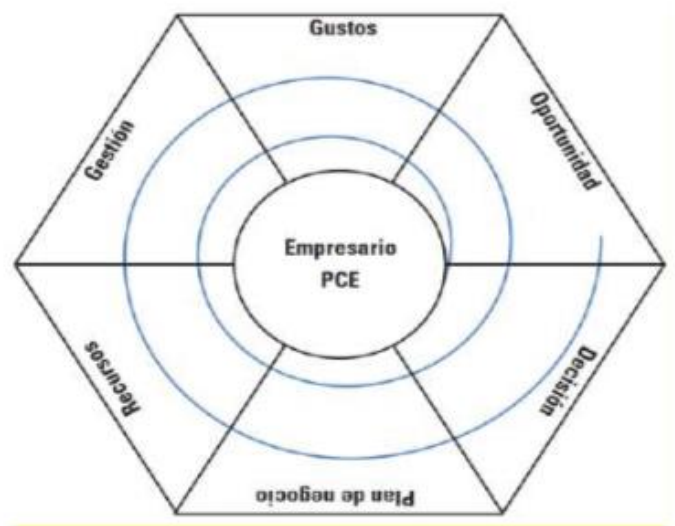

Etapa de recurso: Recursos

Etapa operativa: Gestión

\begin{tabular}{|c|c|c|c|c|}
\hline \multirow{6}{*}{$\begin{array}{c}\text { Shapero y } \\
\text { Sokol (1982) }\end{array}$} & \multirow{6}{*}{$\begin{array}{l}\text { Describen el emprendimiento } \\
\text { como un fenómeno, donde las } \\
\text { intenciones empresariales } \\
\text { dependen de la percepción del } \\
\text { atractivo personal, la } \\
\text { conveniencia personal, la } \\
\text { viabilidad y la propensión a } \\
\text { actuar. }\end{array}$} & \multirow[b]{6}{*}{$\begin{array}{c}\text { Propensión a } \\
\text { actuar }\end{array}$} & $\begin{array}{l}\text { Deseabilidad } \\
\text { Percibida }\end{array}$ & \multirow[b]{6}{*}{$\begin{array}{l}\text { Viabilidad } \\
\text { Percibida }\end{array}$} \\
\hline & & & \multirow{5}{*}{$\begin{array}{l}\text { Intención de } \\
\text { Emprender }\end{array}$} & \\
\hline & & & & \\
\hline & & & & \\
\hline & & & & \\
\hline & & & & \\
\hline \multirow{10}{*}{$\begin{array}{c}\text { Per } \\
\text { Davidsson } \\
(1995)\end{array}$} & Propone un modelo & \multirow{10}{*}{ 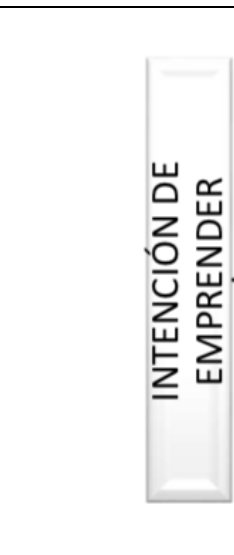 } & \multirow{4}{*}{$\begin{array}{l}\text { Actitudes } \\
\text { Generales }\end{array}$} & \\
\hline & económico - psicológico de & & & \\
\hline & los factores que influyen en & & & \\
\hline & las intenciones de los & & & \\
\hline & individuos para emprender & & \multirow{2}{*}{ Convicciones } & \\
\hline & negocios por si mismos. & & & \\
\hline & Según el modelo las variables & & \multirow{4}{*}{$\begin{array}{c}\text { Actitudes de } \\
\text { dominio }\end{array}$} & \\
\hline & de fondo personal afectan & & & \\
\hline & tanto las actitudes generales & & & \\
\hline & $\begin{array}{l}\text { como las actitudes de } \\
\text { dominio. }\end{array}$ & & & \\
\hline
\end{tabular}




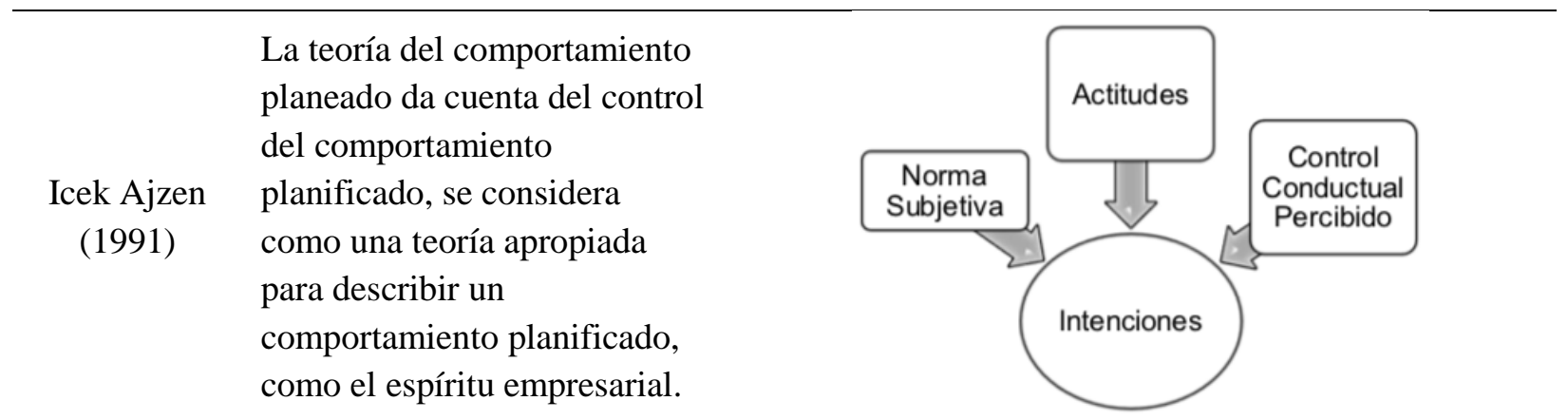

Fuente: elaboración propia con datos de Shapero (1985), McClelland (1961), Gibb (1988), Varela (2008), Shapero y Sokol (1982), Davidsson (1995) y Ajzen (1991).

Para el desarrollo de la investigación que dio origen a este trabajo, se acogieron los postulados de la teoría del comportamiento planeado (TCP) de Ajzen (1991). La TCP es un modelo psicológico que se ha vuelto muy influyente en la investigación empresarial en las últimas décadas (Valencia et al., 2016); esta teoría establece que el comportamiento emprendedor está determinado por las intenciones empresariales. Ajzen (1991) argumenta que es muy probable que un comportamiento dado se realice si existe una fuerte intención de participar en ese comportamiento. La TCP sostiene que la intención depende de la actitud, de la norma subjetiva y del control sobre la conducta que es percibido por el individuo. Este autor establece la premisa de que cualquier comportamiento requiere un cierto nivel de planificación y este puede ser precedido por la intención de adoptar dicho comportamiento. La actitud está compuesta por la creencia y la percepción con relación a su conveniencia personal de ejecutar una determinada acción, que a la vez está relacionada con la expectativa.

De acuerdo con lo anterior, el proceso de tomar la decisión de emprender se puede considerar como una conducta motivada o comportamiento planificado, existiendo una fuerte relación entre las intenciones y la acción. Según la TCP, se supone que las personas son racionales y usan sistemáticamente la información de la que disponen para tomar decisiones. Es una teoría que predice el comportamiento deliberado, porque el comportamiento puede ser planeado.

Según esta teoría la acción humana es conducida por tres clases de consideraciones: a) Actitud hacia el comportamiento, son creencias referidas a las consecuencias probables del comportamiento. Se refiere a la evaluación general de las personas (positiva o negativa) o la evaluación de un comportamiento. Una actitud favorable hacia el comportamiento (comenzar un 
negocio) se forma cuando se percibe que tiene ventajas y consecuencias deseables para ellos. Las consecuencias indeseables resultan de la actitud negativa hacia el comportamiento. b) Normas subjetivas, se refiere a la percepción de los individuos sobre cómo las personas influyentes en sus vidas piensan sobre si participar o no en un comportamiento particular, en este caso iniciar un negocio. La TCP postula que la norma subjetiva o presión social percibida tiene dos componentes: creencias normativas y motivación para cumplir estas creencias. Lo primero se refiere a la probabilidad de que las personas influyentes aprueban o desaprueban el comportamiento de puesta en marcha. El componente de la motivación para cumplir indica la disposición de las personas a cumplir con dichas normas, lo que implica comportarse de acuerdo con las expectativas de otros influyentes. c) Percepción de control del comportamiento, éstas son creencias sobre la presencia de factores que pueden facilitar, o pueden impedir, el desempeño del comportamiento. También se puede definir como la evaluación subjetiva de la propia capacidad y recursos propios que permitan determinar la posibilidad del éxito empresarial.

En cuanto a la educación para emprender, anteriormente predominaba el interés en los estudios de rasgos, que se preocupaban por identificar las diferencias entre las cualidades de los emprendedores. Para los autores Fayolle y Gailly (2008) no existe una receta pedagógica universal sobre cómo enseñar el espíritu empresarial. Aldana-Rivera et al. (2019) indican que el emprendimiento es entendido como una forma de pensar, sentir y actuar para la creación de valor, lo que permite a la comunidad universitaria propiciar espacios para la construcción de conocimientos y el desarrollo de hábitos, actitudes y valores que posteriormente se vean reflejados en acciones orientadas al mejoramiento personal y a la transformación del entorno y de la sociedad. El modelo de la "universidad empresarial" actualmente es reconocido por expertos como un motor importante para el autodesarrollo y la innovación, y como una respuesta adecuada para tener éxito en mercados altamente turbulentos e impredecibles (Hannon, 2013). La universidad emprendedora es una universidad que monitorea de cerca lo que está sucediendo en el medio ambiente y responde fácilmente a los cambios y señales de ese ambiente. La discusión sobre la efectividad de la educación emprendedora está vinculada a un debate importante, todavía en curso (Sánchez et al., 2017). El debate gira en torno a aspectos como si el emprendimiento implica individuos con una personalidad definida o con habilidades desarrollables (Bechard y Toulouse, 1998). 
Por último, se presentan los sectores de clase mundial identificados por la Fiduciaria Colombiana de Comercio Exterior S.A. (FIDUCOLDEX, 2017), con la creación del programa de transformación productiva impulsado por el gobierno nacional. El gobierno colombiano pretende impulsar 20 sectores de la economía nacional para convertirlos en sectores de clase mundial, competitivos y altamente productivos; los 20 sectores, se dividen en tres grandes grupos: Manufacturas, Agroindustria y Servicios. Para entender la importancia de cómo los futuros profesionales se pueden involucrar con sus iniciativas emprendedoras en el fortalecimiento de estos sectores, se enlistan algunos de los retos que están pendientes por afrontar: desarrollo de modelos de importación, innovación, intensificación en la utilización de TIC, subfacturación, formalización del empleo, apertura a mercados internacionales, desarrollo de nuevos productos, mejora en las técnicas productivas agropecuarias, formación de capital humano, actualización de modelos de negocio, entre otros aspectos. Para el presente estudio serán objeto de análisis los estudiantes de carreras profesionales en áreas tales como: Ciencias Económicas y Administrativas, Ciencias de la Salud, Ingenierías, Agroindustria, Industria Gráfica, y Ciencias Ambientales y Ciencias Pecuarias (Ver Tabla 2).

\section{Tabla 2}

Programas de la Universidad de Nariño relacionados con los sectores de clase mundial

\begin{tabular}{lll}
\hline \multicolumn{1}{c}{ Manufactura } & \multicolumn{1}{c}{ Agroindustria } & \multicolumn{1}{c}{ Servicios } \\
\hline Diseño Industrial & Biología & Diseño Gráfico \\
& Ing. Agronómica & Administración de Empresas \\
Ing. Ambiental & Contaduría Pública \\
Ing. Agroforestal & Economía \\
Ing. Agroindustrial & Mercadeo \\
& Ing. de Producción Acuícola & Comercio Internacional \\
& Medicina Veterinaria & Medicina \\
& Zootecnia & Ing. Civil \\
& Química & Ing. Electrónica \\
& Ing. de Sistemas \\
& & Psicología \\
\hline
\end{tabular}

Fuente: elaboración propia a partir de Ruiz (2014). 


\section{Metodología}

La investigación que dio origen a este artículo es exploratoria y descriptiva. De acuerdo con Hernández et al. (2014), en la investigación descriptiva se busca especificar las propiedades, las características y los perfiles de personas, grupos, procesos, objetos o cualquier otro fenómeno que se someta a un análisis. Los mismos autores afirman que los estudios exploratorios se emplean cuando el tema o problema de investigación ha sido poco estudiado, como es el caso de la intención emprendedora en los estudiantes de la Universidad de Nariño. Con esta investigación se pretende explorar y describir cuales son las creencias de comportamiento, normativas y controladas de los estudiantes de pregrado de la Universidad de Nariño, que cursan carreras profesionales relacionadas con los sectores de clase mundial, y cómo estas creencias influyen en la intención emprendedora.

En cuanto a la población y muestra definidas para la investigación, según la Oficina de Registro y Control Académico, de la Universidad de Nariño, en el periodo B de 2017, se encontraban matriculados un total 1.219 estudiantes en los dos últimos semestres (noveno y décimo) de los programas relacionados con los sectores de Clase Mundial, que se definieron en el apartado anterior. Teniendo el dato de la población, se procede al cálculo de la muestra de la siguiente manera:
$n=\quad \underline{N * Z 2 * P * Q}$
Dónde:
$\mathrm{N}: 1.219$
$(N-1) * e 2+Z 2 * P * Q$
Z: 1,96
P: 0,5
Q: 0,5
e: 0,05

En consecuencia, el tamaño muestral es 293; es decir que se aplicaron encuestas a 293 estudiantes. Se puede afirmar que la muestra es representativa y confiable porque el nivel de confianza es del 95\%; el indicador de fiabilidad del estudio y de la exactitud de los resultados (margen de error) es del 5\%; y el dato de la población fue obtenido de una fuente oficial de la universidad. La aplicación de las encuestas se realizó de manera presencial en los salones de clase, en el semestre A de 2018. 
Además de lo anterior, se entrevistaron 21 de directores de los programas seleccionados y los 9 Decanos de las Facultades que acogen estos programas, esto con el fin de conocer la percepción sobre el emprendimiento, de las personas que toman las decisiones con respecto al perfil profesional de los estudiantes.

\section{Resultados}

A continuación, se presentan los resultados del análisis de la intención emprendedora de los estudiantes de pregrado de la Universidad de Nariño, desde las creencias de comportamiento, las creencias normativas y las creencias controladas planteadas por Ajzen (1991). Se debe tener en cuenta la Tabla 3 para la interpretación de los resultados.

\section{Tabla 3}

Identificación de las variables

\begin{tabular}{|c|c|c|c|}
\hline Categorías & Subcategorías & Variables & Siglas \\
\hline \multirow{9}{*}{$\begin{array}{c}\text { Creencias de } \\
\text { Comportamiento }\end{array}$} & \multirow{9}{*}{$\begin{array}{l}\text { Actitudes positivas } \\
\text { para emprender }\end{array}$} & He pensado seriamente en iniciar una empresa & AP1 \\
\hline & & Haré todo lo posible por iniciar y dirigir mi propia & AP? \\
\hline & & Identificar y superar un reto personal & AP3 \\
\hline & & Posibilidad de ganar más dinero que si fuera empleado & AP4 \\
\hline & & $\begin{array}{l}\text { Ser mi propio jefe, manejar mi tiempo y alcanzar la } \\
\text { independencia }\end{array}$ & A \\
\hline & & Lograr mi autorrealización y satisfacción personal & AP6 \\
\hline & & Dificultad para conseguir empleo & AP7 \\
\hline & & En su plan de vida no se visualiza como empleado & AP8 \\
\hline & & $\begin{array}{l}\text { La alta posición social que ocupan quienes son } \\
\text { empresarios }\end{array}$ & AP9 \\
\hline \multirow{7}{*}{$\begin{array}{l}\text { Creencias } \\
\text { Normativas }\end{array}$} & \multirow{7}{*}{$\begin{array}{l}\text { El contexto } \\
\text { universitario }\end{array}$} & $\begin{array}{l}\text { He asistido al menos a un curso de emprendimiento } \\
\text { como espacio obligatorio de mis estudios }\end{array}$ & CU1 \\
\hline & & $\begin{array}{l}\text { He asistido al menos a un curso de emprendimiento } \\
\text { como espacio electivo de mis estudios }\end{array}$ & CU2 \\
\hline & & $\begin{array}{l}\text { Estoy estudiando un programa específico de } \\
\text { emprendimiento por mi cuenta }\end{array}$ & CU3 \\
\hline & & $\begin{array}{l}\text { No he asistido a un curso de emprendimiento hasta } \\
\text { ahora }\end{array}$ & CU4 \\
\hline & & $\begin{array}{l}\text { El entorno de mi universidad me inspira a desarrollar } \\
\text { ideas para nuevos negocios }\end{array}$ & CU5 \\
\hline & & $\begin{array}{l}\text { En mi universidad los estudiantes son motivados para } \\
\text { involucrarse en actividades emprendedoras }\end{array}$ & CU6 \\
\hline & & $\begin{array}{l}\text { Las materias de emprendimiento de la universidad } \\
\text { desarrollan mi intención de crear una empresa }\end{array}$ & CU7 \\
\hline
\end{tabular}




\begin{tabular}{|c|c|c|c|}
\hline & & $\begin{array}{l}\text { La universidad proporciona los conocimientos } \\
\text { necesarios para ser profesional más que para ser } \\
\text { empresario }\end{array}$ & CU8 \\
\hline & \multirow{3}{*}{ El contexto familiar } & Mis padres me motivan a ser emprendedor & CF1 \\
\hline & & Mis familiares cercanos me motivan a ser emprendedor & CF2 \\
\hline & & $\begin{array}{l}\text { Alguno de los miembros de mi familia tiene un negocio } \\
\text { o empresa propia }\end{array}$ & CF3 \\
\hline & \multirow{8}{*}{$\begin{array}{l}\text { El contexto social y } \\
\text { cultural }\end{array}$} & A tus amigos les interesa emprender & CSC1 \\
\hline & & Alguno de tus amigos es emprendedor & CSC2 \\
\hline & & $\begin{array}{l}\text { Es mejor ser dueño de su propia empresa que empleado } \\
\text { en una ajena }\end{array}$ & CSC3 \\
\hline & & $\begin{array}{l}\text { En esta región se admira a quienes dirigen sus propias } \\
\text { empresas }\end{array}$ & CSC4 \\
\hline & & $\begin{array}{l}\text { Tener una empresa propia genera poder, respeto y un } \\
\text { estatus social alto }\end{array}$ & CSC5 \\
\hline & & $\begin{array}{l}\text { Algunas creencias religiosas castigan a los ricos y } \\
\text { promueven la pobreza }\end{array}$ & CSC6 \\
\hline & & Baja confianza en mis capacidades emprendedoras & CSC7 \\
\hline & & No tengo claro qué empresa quisiera crear & CSC8 \\
\hline \multirow{6}{*}{$\begin{array}{l}\text { Creencias } \\
\text { Controladas }\end{array}$} & \multirow{6}{*}{$\begin{array}{l}\text { Obstáculos para } \\
\text { crear empresa }\end{array}$} & Escaso capital para iniciar la empresa & OCE1 \\
\hline & & Muchos impuestos y trámites para crear una empresa & OCE2 \\
\hline & & Insuficiente apoyo de entidades públicas y privadas & OCF3 \\
\hline & & Mi carrera universitaria no es para crear empresa & OCE4 \\
\hline & & Falta de socios para iniciar una empresa & OCE5 \\
\hline & & No sé producir ni fabricar nada en particular & OCE6 \\
\hline
\end{tabular}

Fuente: elaboración propia

\section{Creencias de comportamiento que influyen en la intención emprendedora de los estudiantes}

En la Figura 1 se observa que la mayoría de los estudiantes pertenecientes al grupo objeto de estudio, están totalmente de acuerdo y de acuerdo (70\%), con la idea de iniciar una empresa (AP1). En lo que respecta a iniciar y dirigir su propia empresa en el futuro (AP2), se evidencia que sí tienen esta intención, ya que el $73 \%$ de las respuestas son positivas. En cuanto a si ven el emprendimiento como la forma de identificar y superar un reto personal (AP3), están totalmente de acuerdo y de acuerdo con esta afirmación el $73 \%$ de los estudiantes. En lo concerniente a las ventajas económicas que pueden derivarse de ser dueños de su propia empresa y ganar más dinero que como empleados (AP4), el 78\% de las respuestas son positivas. En cuanto a ser su propio jefe, manejar su tiempo personal y alcanzar la independencia (AP5), el $84 \%$ de las respuestas son positivas. Se puede decir que existe una orientación favorable por parte de los estudiantes, para la creación de empresas, porque perciben la posibilidad de tener independencia financiera y de poder 
orientar el uso del tiempo personal. Estos resultados coinciden con lo planteado por GalleguillosCortés et al. (2019), quienes en un estudio sobre predictores de la intención emprendedora en alumnos de pregrado, determinan que las creencias individuales sobre el emprendimiento pueden influir en la intención emprendedora.

\section{Figura 1}

Actitudes positivas para emprender de los estudiantes de la Universidad de Nariño

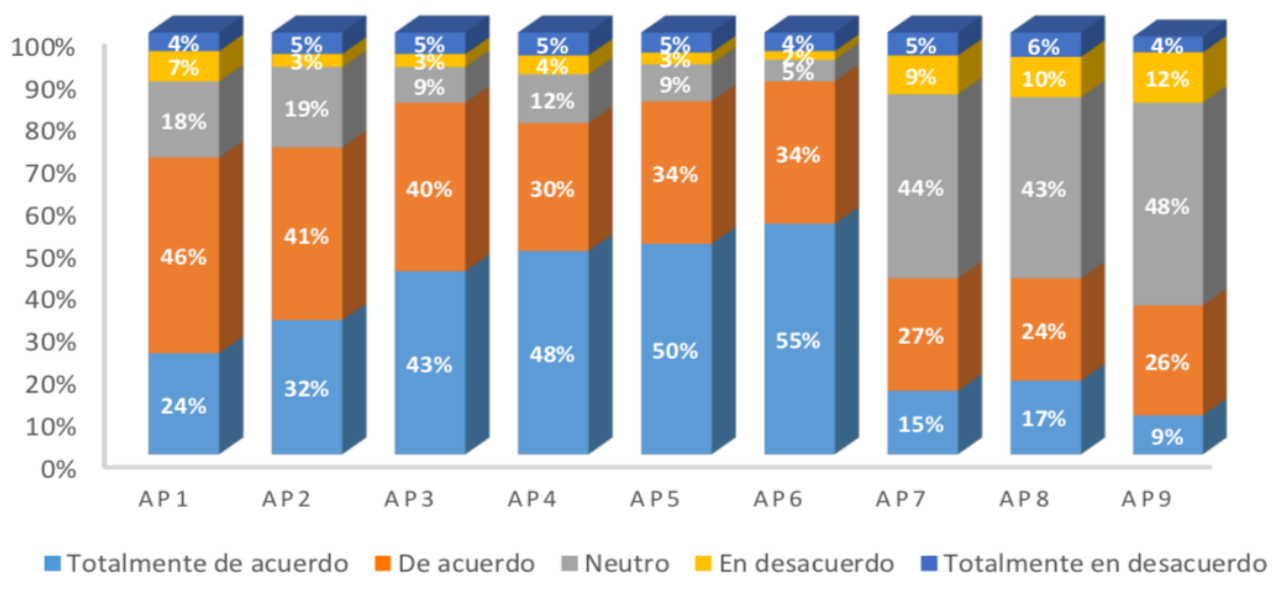

Fuente: elaboración propia.

En cuanto a lograr su autorrealización y satisfacción personal (AP6), el 89\% de las respuestas son positivas, los estudiantes consideran que iniciar un emprendimiento ayudaría a lograr su autorrealización. Con relación a emprender porque existe dificultad para conseguir empleo (AP7), el $44 \%$ de las respuestas son neutras y el $42 \%$ positivas, la mayoría de los estudiantes prefiere no opinar frente al tema; sin embargo, otro grupo significativo está de acuerdo con esta proposición y ven en el emprendimiento una posibilidad para emplearse. En lo que respecta a si el estudiante no se visualiza como empleado (AP8), el 44\% de las respuestas son neutras y el $41 \%$ positivas, se evidencian porcentajes similares a los de la variable anterior, y a los de la siguiente (AP9), ya que el $48 \%$ de respuestas son neutras y 35\% positivas, cuando se hace referencia a la alta posición social que ocupan quienes son empresarios. Los estudiantes consideran el emprendimiento como un factor relevante para su desarrollo personal, sin embargo, la mayoría mantiene una posición neutra cuando se les pregunta si se visualizan a futuro como empleados o emprendedores. En síntesis, se puede afirmar que un porcentaje considerable de los estudiantes de la Universidad de 
Nariño, tienen una apreciación positiva respecto a las variables relacionadas con las creencias de comportamiento que inciden en la intención emprendedora, pero llama la atención que un mayor porcentaje de respuestas relacionadas con las tres últimas variables son neutras.

\section{Creencias normativas del entorno cercano al estudiante que inciden en el desarrollo del espíritu emprendedor}

Como se puede observar en la Figura 2, en lo que se refiere a la asistencia a espacios obligatorios de emprendimiento en el contexto universitario (CU1), el 50\% de respuestas son positivas, es decir, que la mitad de los estudiantes que participaron en el estudio validan los espacios de emprendimiento generados por la Universidad. En relación a si han asistido al menos a un curso de emprendimiento como espacio electivo de sus estudios (CU2), el 35\% de las respuestas son positivas, el $35 \%$ negativas y el $27 \%$ neutras, en este caso no se evidencia que la mayoría de los estudiantes tengan una orientación positiva a tomar cursos electivos de emprendimiento.

\section{Figura 2}

Factores del contexto universitario que influyen en la intención emprendedora de los estudiantes

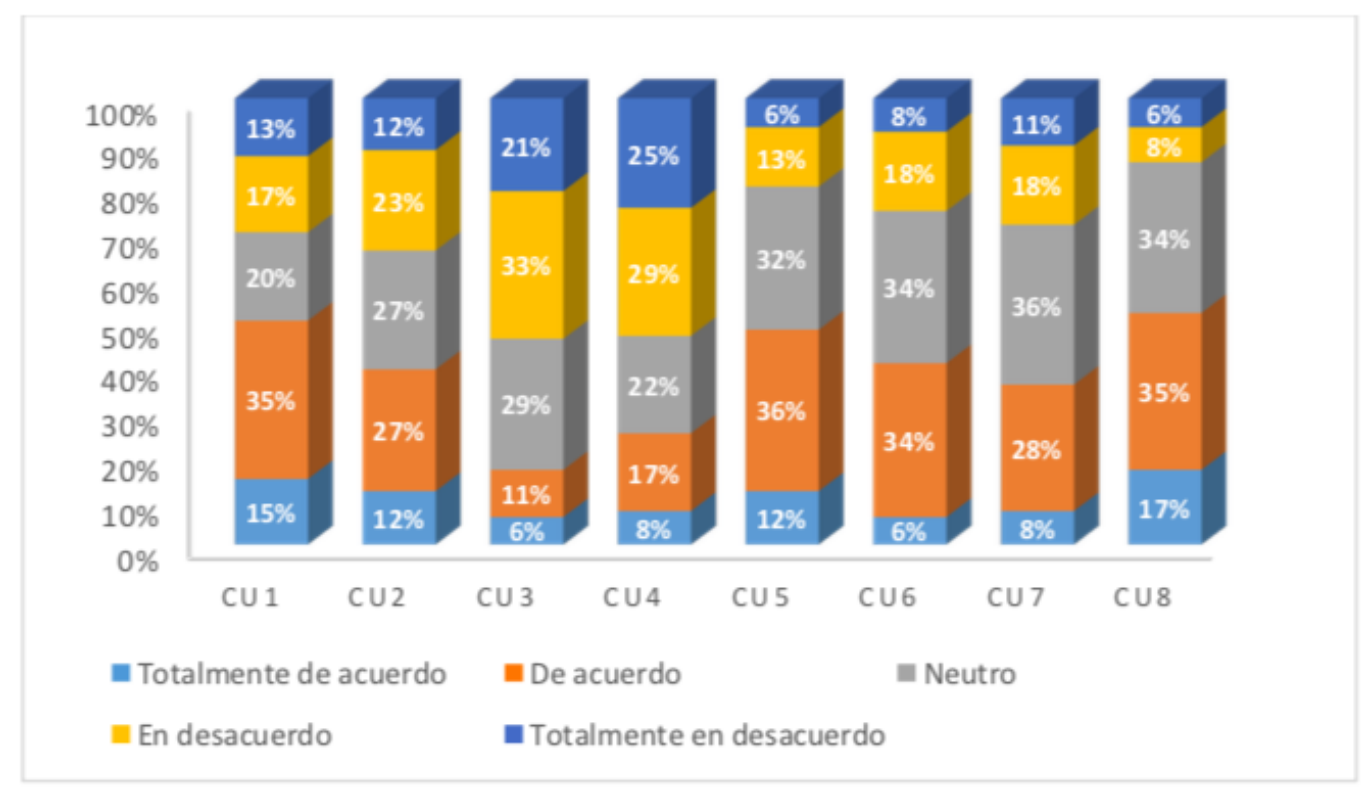

Fuente: elaboración propia. 
Con respecto a si estudian un programa específico de emprendimiento por su cuenta (CU3), el $54 \%$ de las respuestas son negativas y el 29\% neutras; la mayoría de los estudiantes manifestó que no asiste, por iniciativa personal, a algún programa de formación específica en emprendimiento. El 54\% de los estudiantes manifiesta que no ha asistido a un curso de emprendimiento hasta el momento (CU4). Se puede apreciar que en la mayoría de los estudiantes objeto de estudio no existe interés por tomar cursos relacionados con el emprendimiento en la universidad, y aquellos que si lo hacen, pertenecen a programas en los que reciben emprendimiento como parte obligatoria de su formación. Frente a la premisa de que la universidad inspira a desarrollar ideas para nuevos negocios (CU5), el 48\% de las respuestas son positivas y el 32\% neutras; la mayoría de los estudiantes están de acuerdo con esta afirmación y ven a la universidad como un lugar inspirador, otros por el contrario prefieren no opinar.

Frente a la pregunta de que si en la universidad los estudiantes son motivados para involucrarse en actividades emprendedoras (CU6), el $40 \%$ de las respuestas son positivas y $34 \%$ neutras. Con respecto a si las materias de emprendimiento de la universidad desarrollan la intención de crear una empresa (CU7), el 36\% de las respuestas son positivas y el 36\% neutras; la opinión se encuentra dividida entre aquellos que si están de acuerdo y los que no opinan sobre el tema. En cuanto a si la universidad proporciona los conocimientos necesarios para ser profesional más que para ser empresario (CU8), el 52\% de los estudiantes está de acuerdo con la afirmación y el 34\% de las respuestas son neutras. Para los estudiantes es positivo que la universidad les proporcione los conocimientos necesarios para ser buenos profesionales.

Con respecto a las variables evaluadas en este apartado, existen aspectos que si inciden en la intención emprendedora, derivados del contexto universitario, como los cursos obligatorios y electivos que se ofrecen sobre esta temática. Sin embargo, frente a las creencias normativas del entorno universitario, se puede notar mayoritariamente una posición neutral.

Otros factores importantes que pueden influenciar la intención emprendedora de los estudiantes pertenecen al contexto familiar (ver Figura 3). Entre los factores relacionados con el ámbito familiar, se preguntó si los padres motivan a ser emprendedor (CF1), el 80\% de respuestas fueron positivas y el $18 \%$ neutras. De igual forma se puede ver que los estudiantes consideran que la 
familia en general es motivadora a la hora de emprender (CF2), el $61 \%$ de las respuestas son positivas y $28 \%$ neutras. Los estudiantes reconocen en su entorno próximo personas que tienen un negocio o empresa propia (CF3), con respecto a esta premisa el $60 \%$ de las respuestas son positivas. Se puede decir que en algún momento, los familiares cercanos pueden servir como ejemplo y modelo a seguir, el acercamiento de los estudiantes a los negocios familiares puede motivarlos a emprender. En cuanto al contexto familiar, los resultados coinciden con el estudio de Galleguillos-Cortés et al. (2019), quienes concluyen que las creencias sociales (normativas) pueden influir en la intención emprendedora

\section{Figura 3}

Factores del contexto familiar que influyen en la intención emprendedora de los estudiantes

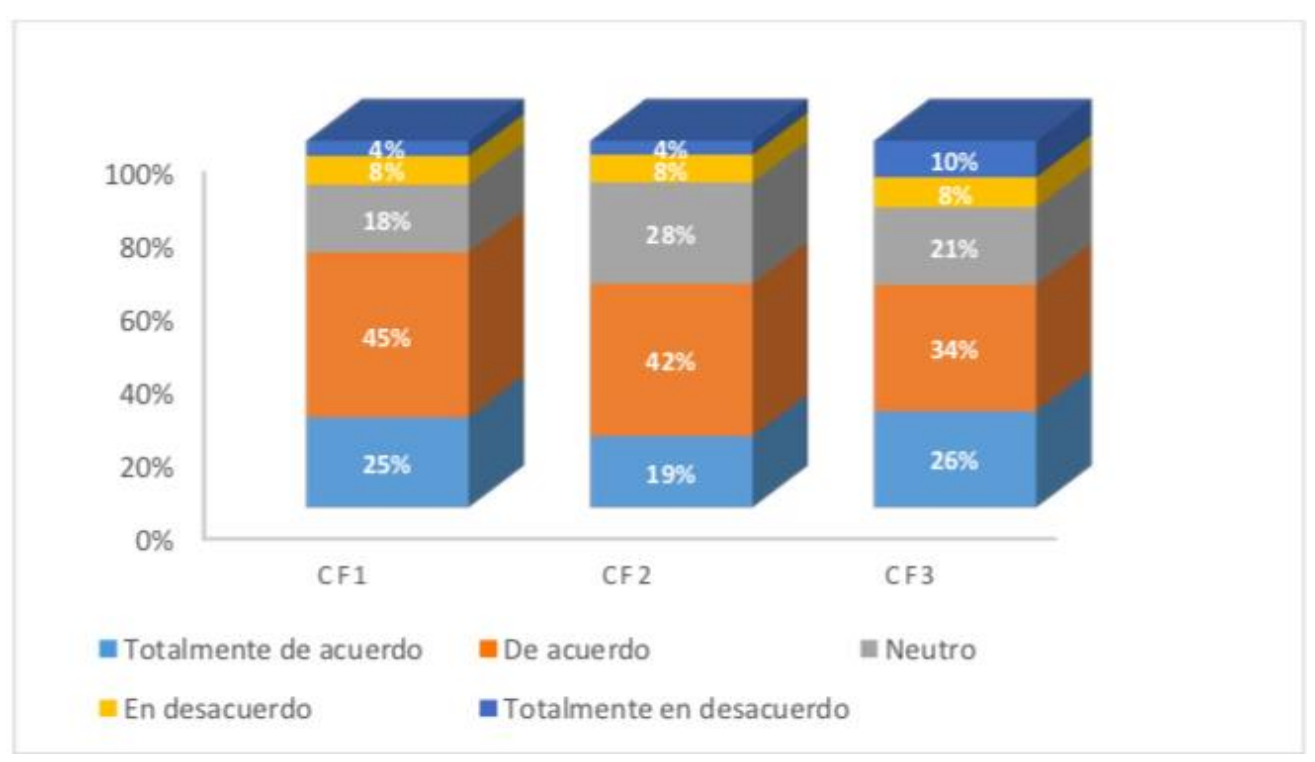

Fuente: elaboración propia.

Además de lo anterior, el contexto social y cultural también puede ejercer influencia en la intención emprendedora de los estudiantes. En este apartado se analiza el contexto que rodea al estudiante, distinto al familiar, más bien el contexto social y cultural en el que se ve inmerso por ser parte de una comunidad o una región (ver Figura 4). En cuanto a la pregunta de si a sus amigos les interesa emprender (CSC1), el 55\% de las respuestas son positivas y el 38\% neutras; y cuando se indagó si alguno de sus amigos es emprendedor (CSC2), el $60 \%$ de las respuestas son positivas y $28 \%$ neutras. 


\section{Figura 4}

Factores del contexto social y cultural que influyen en la intención emprendedora de los estudiantes

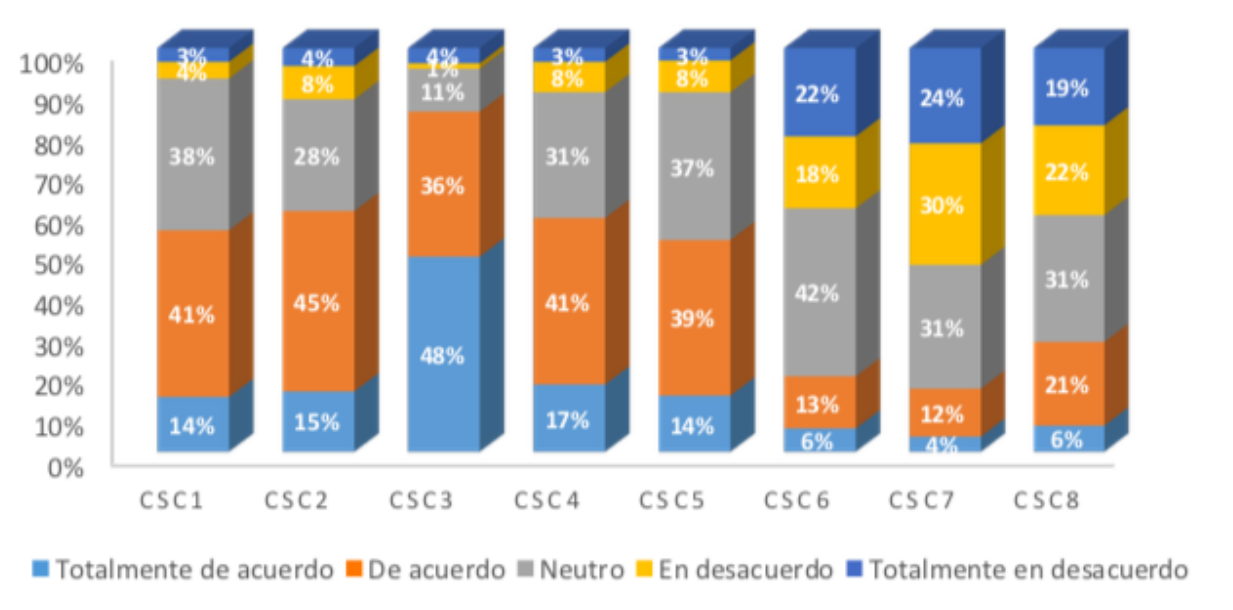

Fuente: elaboración propia.

Se evidencia que los estudiantes ven como una mejor opción ser dueños de su propia empresa que ser empleados (CSC3), ya que el 84\% de las respuestas son positivas. En lo referente a si en esta región se admira a quienes dirigen sus propias empresas (CSC4), el 58\% de las respuestas son positivas y el $31 \%$ neutras. Cuando se pregunta si tener una empresa propia genera poder, respeto y un estatus social alto (CSC5), el 53\% de las respuestas son positivas y el 37\% neutras. Cuando se indagó con respecto a si algunas creencias religiosas castigan a los ricos y promueven la pobreza (CSC6), el $40 \%$ de respuestas son negativas y el $42 \%$ neutras, parece que a la hora de hablar de religión muchos prefieren mantener una postura neutral, y otro porcentaje no está de acuerdo con esa afirmación.

En lo que tiene que ver con si la baja confianza en sus capacidades emprendedoras incide en la intención de emprender (CSC7), el 54\% de respuestas son negativas y el 31\% neutras, es decir que los estudiantes no consideran que tengan bajas capacidades y que esto llegue a influir al momento de tener una intención emprendedora. Por último, cuando se preguntó sí no tiene claro qué empresa quisieran crear (CSC8), el 41\% de las respuestas son positivas y el $31 \%$ neutras; un alto porcentaje de los estudiantes no tiene claro qué tipo de empresa tendrían en el futuro y otro porcentaje prefiere no opinar sobre el tema. 
Frente a las variables que hacen parte del contexto social y cultural, se puede establecer que si son determinantes para la intención emprendedora, pero se observa un alto porcentaje de neutralidad en todas las respuestas. Tarapuez-Chamorro et al. (2019), realizaron un estudio de la intensión emprendedora en estudiantes de Maestrías en Administración en Colombia, y concluyeron que entre los factores que determinan la intención emprendedora en estos estudiantes, se encuentran dos relacionados con creencias normativas del contexto social y cultural: alguno de tus amigos es emprendedor y tener una empresa propia genera poder, respeto y un estatus social alto. En el presente estudio se obtuvieron resultados similares, ya que el $60 \%$ de las respuestas fueron positivas cuando se indago respecto a si alguno de tus amigos es emprendedor y el 53\% de las respuestas fueron positivas cuando se preguntó si tener una empresa propia genera poder, respeto y un estatus social alto; llama la atención que un $28 \%$ de las respuestas para la primera pregunta y un $37 \%$ para la segunda pregunta son neutras.

\section{Creencias controladas que influyen en las capacidades emprendedoras de los estudiantes}

Por último, se abordan las creencias controladas que influyen en las capacidades emprendedoras de los estudiantes de la Universidad de Nariño, teniendo en cuenta los obstáculos que son percibidos por los estudiantes (Figura 5) y las razones que inciden en la generación de ideas de negocio.

\section{Figura 5}

Obstáculos para crear empresa que son percibidos por los estudiantes

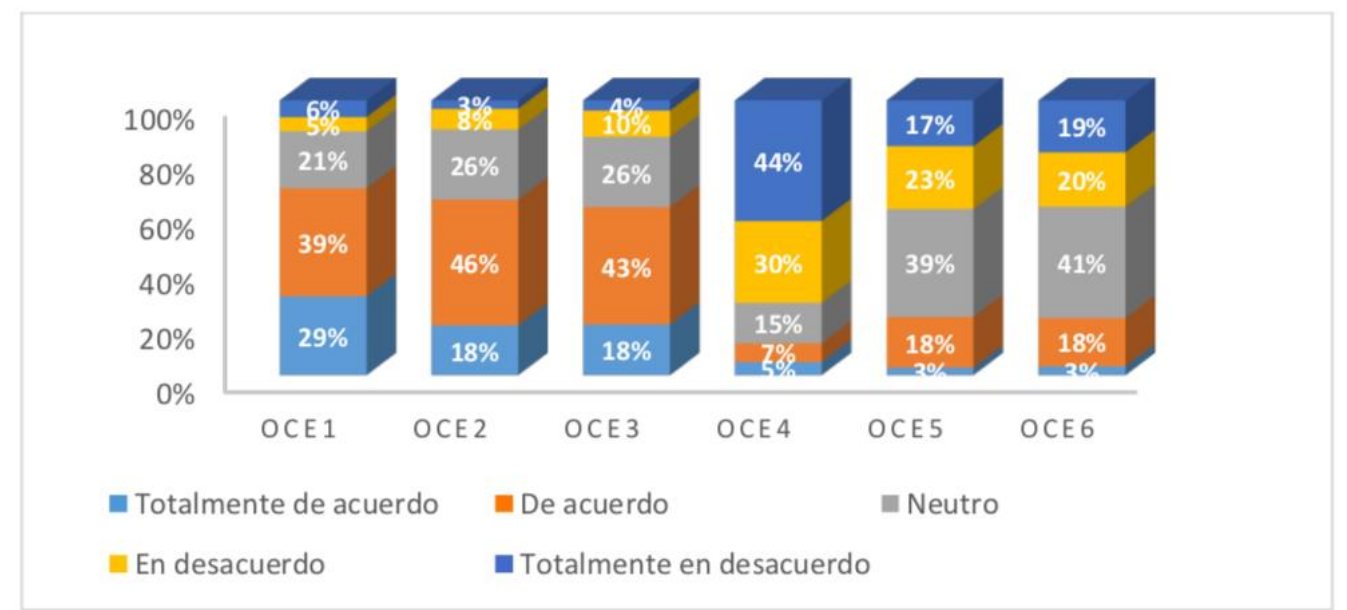

Fuente: elaboración propia. 
Cuando se interroga a los estudiantes respecto a los obstáculos que tienen que ver con la intención emprendedora, se tiene que el escaso capital para iniciar la empresa (OCE1), obtiene un 68\% de respuestas positivas, es decir que consideran este aspecto como limitante a la hora de emprender; en cuanto a la premisa de si existen muchos impuestos y trámites para crear una empresa (OCE2), el 64\% de respuestas son positivas; cuando se indaga sobre el insuficiente apoyo de entidades públicas y privadas para crear la empresa (OCE3), el 61\% de las respuestas son positivas, esto quiere decir que un alto porcentaje de los estudiantes no perciben facilidades para conseguir la financiación necesaria para emprender y consideran que existen muchos trámites para crear empresa.

En cuanto a la premisa sobre si su carrera universitaria no es para crear empresa (OCE4), el 74\% de las respuestas son negativas, no lo ven de esa forma, por el contrario, la carrea se mira como un gran potencial para crear empresa. Cuando se pregunta por la falta de socios para iniciar una empresa (OCE5), el 40\% de las respuestas son negativas y el 39\% neutras, en este tema algunos prefieren no opinar, pero la mayoría no creen que la falta de socios sea determinante a la hora de querer emprender. En lo que respecta a si no sabe producir o fabricar algo en particular (OCE6), el 39\% de los estudiantes están totalmente en desacuerdo y en desacuerdo con esta premisa y el $41 \%$ de las respuestas son neutras. Se puede decir entonces que las variables analizadas no son vistas como obstáculos para emprender, sin embargo, existe una tendencia en los estudiantes a no opinar sobre estos temas. En el caso de las creencias controladas, este estudio presenta resultados similares al realizado por Tarapuez-Chamorro et al. (2019), en las respuestas a las últimas tres variables, ya que el mayor porcentaje de las respuestas para estas preguntas son negativas, es decir que los estudiantes no ven como limitantes para sus capacidades emprendedores que su carrera universitaria no sea para crear empresa, un alto porcentaje (74\%), así lo cree; la falta de socios para iniciar una empresa o el no saber producir o fabricar algo (en los dos últimos casos llama la atención el elevado porcentaje de respuestas neutras).

Finalmente, en cuanto a las entrevistas realizadas a los directivos universitarios, los factores que más se repiten, según en el tipo de creencia son: (1) Creencias de comportamiento: honestidad, capacidad de trabajo, gente muy valiosa que ha luchado contra las adversidades y también la perseverancia del nariñense, (2) Creencias de normativas: la situación económica de Colombia, 
ausencia de verdaderos estímulos para generar empresas y la motivar a los estudiantes para que conozcan los diferentes programas que tiene la región o el Gobierno Nacional para la creación de empresa, por ejemplo, el Fondo Emprender y las diferentes acciones del Ministerio de Industria y Comercio. (3) Creencias Controladas: lo que se busca son negocios muy tradicionales, el Estado no protege la producción nacional y hay carencia en cuanto a vías terciarias. De acuerdo con lo anterior los entrevistados proponen algunas estrategias de mejoramiento: desarrollar una escuela de formadores empresarios; ofrecer preparación psicológica, motivacional y diversificar la formación los estudiantes; que los estudiantes conozcan de emprendimiento, de empresarismo, de finanzas, de formulación y evaluación de proyectos y de matemáticas financieras, entre otros temas importantes.

\section{Conclusiones}

En cuanto a las creencias de comportamiento, relacionadas con las actitudes positivas para emprender, las que sobresalen en los estudiantes de la Universidad de Nariño (con un porcentaje de aceptación por encima del 75\%) son: las ventajas económicas que pueden derivarse de ser dueños de su propia empresa y ganar más dinero que como empleados; ser su propio jefe y manejar su tiempo personal; y lograr su autorrealización y satisfacción personal. Estas actitudes están determinadas por las creencias de que cierto comportamiento conducirá a un resultado favorable; en este caso particular se perciben como importantes para los estudiantes de la Universidad de Nariño, la de independencia financiera y laboral, el manejo del tiempo y la autorrealización. Llama la atención que ante variables como: la dificultad para conseguir empleo, en su plan de vida no se visualiza como empleado y la alta posición que ocupan quienes son empresarios, más del $40 \%$ de las respuestas son neutras, y por debajo de este porcentaje están las respuestas positivas. En este caso se podría decir que no predominan actitudes positivas para emprender en relación con estas tres variables.

En lo que respecta a las creencias normativas, las cuales miden el valor que las personas asignan a la opinión de otras personas cercanas a ellos; en los estudiantes de la Universidad de Nariño sobresalen en las relacionadas con el contexto universitario: la universidad proporciona los conocimientos necesarios para ser profesional más que para ser empresario, $52 \%$ de los estudiantes 
está de acuerdo con esta proposición; el 54\% de los estudiantes está totalmente en desacuerdo y en desacuerdo con los enunciados de si está estudiando un programa específico de emprendimiento por su cuenta, y que no ha asistido a un curso de emprendimiento hasta ahora. De acuerdo con estos resultados, los estudiantes consideran positivo que la universidad les brinde los conocimientos para ser buenos profesionales, pero el contexto universitario no los orienta a emprender.

En las creencias normativas relacionadas con el contexto familiar sobresalen: mis padres me motivan a ser emprendedor, el $80 \%$ de los estudiantes están totalmente de acuerdo y de acuerdo con esta afirmación; alguno de los miembros de mi familia tiene un negocio o empresa propia, el $70 \%$ de los estudiantes está totalmente de acuerdo y de acuerdo con esta premisa; y mis familiares cercanos me motivan a ser emprendedor, el $61 \%$ de los estudiantes están totalmente de acuerdo y de acuerdo con esta afirmación. Se puede afirmar que el contexto familiar es importante para que los estudiantes de la Universidad de Nariño emprendan.

Y en las creencias normativas relacionadas con el contexto social y cultural sobresalen: es mejor ser dueño de su propia empresa que ser empleado en una ajena, con $80 \%$ de estudiantes totalmente de acuerdo y de acuerdo; y alguno de tus amigos es emprendedor con un $60 \%$ de estudiantes totalmente de acuerdo y de acuerdo. Estas variables del contexto social son importantes para los estudiantes de la Universidad de Nariño a la hora de crear empresa. Otro aspecto a resaltar es que el $54 \%$ y el $41 \%$ de los estudiantes respectivamente están totalmente en desacuerdo y en desacuerdo con relación a las premisas baja confianza en mis capacidades emprendedoras y no tengo claro que empresa quisiera crear; así mismo se observa un alto porcentaje de respuestas neutras relacionadas con estas dos premisas. Lo anterior indica que algunas variables del contexto social y cultural son importantes en la intención emprendedora de los estudiantes de la Universidad de Nariño, y que tienen confianza en sus capacidades y no ven como limitante no tener claro que empresa quieren crear.

En relación con las creencias controladas, relacionadas con los obstáculos para crear empresa, las que sobresalen en los estudiantes de la Universidad de Nariño son: escaso capital para iniciar la empresa, con $68 \%$ de estudiantes totalmente de acuerdo y de acuerdo, y muchos impuestos y 
trámites para crear una empresa con $64 \%$ de respuestas positivas; estas percepciones pueden influir negativamente en la intención emprendedora de los estudiantes, ya que se asocian con la dificultad de iniciar un comportamiento positivo que lleve a emprender. En cuanto a la premisa de que su carrera universitaria no es para crear empresa, el 74\% de los estudiantes están en desacuerdo con esta premisa, lo que significa que no consideran que sea un obstáculo para emprender. Por último, en cuanto a la falta de socios para una iniciar una empresa y no sé producir ni fabricar nada en particular, cerca del $40 \%$ de los estudiantes en los dos casos está totalmente en desacuerdo y en desacuerdo con esta afirmación, esto quiere decir que no ven estos aspectos como limitantes para crear empresa. Llama la atención que cerca del $40 \%$ de los estudiantes frente a las dos premisas anteriores tiene una posición neutra. En estos casos la percepción de los estudiantes no refleja un comportamiento controlable hacia la posibilidad de emprender.

A partir de este trabajo, se podría establecer a futuro, una ruta para fortalecer el programa de formación para el emprendimiento en los estudiantes de la Universidad, a través de la unidad de emprendimiento denominada Universidad de Nariño Empresarial - UNEMPRESA. Lo anterior haciendo énfasis en los aspectos percibidos por los estudiantes y las estrategias propuestas por los directivos. La ruta sería un espacio creado por la Universidad, para que aquellos estudiantes que desean emprender, tengan un acompañamiento institucional desde la etapa de ideación hasta la puesta en marcha de los emprendimientos. Esta propuesta debe estar acompañada de una política institucional que priorice el emprendimiento y adicionalmente, se sugiere la implementación de una cátedra transversal en emprendimiento para todos los programas de la Universidad.

\section{Referencias}

(1) Ajzen, I. (1991). The theory of planned behavior. Organizational Behavior and Human Decision Processes, 50(2), 179-211. https://doi.org/10.1016/0749-5978(91)90020-T

(2) Aldana-Rivera, E. E., Tafur-Castillo, J., Gil, I., \& Mejía, C. (2019). Práctica pedagógica de emprendimiento en docentes de educación superior en Institución Educativa Universitaria de Barranquia. Archivos Venezolanos de Farmacología y Terapéutica, 38(2), 9-22. http://www.redalyc.org/articulo.oa?id=55964524002 
(3) Azqueta, A. (2017). El concepto de emprendedor: origen, evolución e introducción. Simposio Internacional El Desafío de Emprender en la Escuela del Siglo XXI, Sevilla, España. https://hdl.handle.net/11441/74177

(4) Baggozi, R., Baumgartner, J., \& Yi, Y. (1989). An investigation into the role of intentions as mediators of the attitude-behavior relationship. Journal of Economic Psychology, 10(1), 3562. https://doi.org/10.1016/0167-4870(89)90056-1

(5) Baron, R. A. (2007). Behavioral and cognitive factors in entrepreneurship: Entrepreneurs as the active element in new venture creation. Strategic Entrepreneurship Journal, 1(1-2), 167182. https://doi.org/10.1002/sej.12

(6) Bechard, J., \& Toulouse, J. (1998). Validation of the didactic model for the analysis of training objectives in entrepreneurship. Journal of Business Venturing, 13(4), 317-332. https://doi.org/10.1016/S0883-9026(98)80006-2

(7) Bird, B. (1988). Implementing entrepreneurial ideas: the case for intention. Academy of Management Review, 13(3), 442-453. https://doi.org/10.5465/amr.1988.4306970

(8) Davidsson, P. (1995). Determinants of entrepreneurial intentions. RENT IX Workshop, 1-31.

(9) Durán-Aponte, E. y Arias-Gómez, D. (2015). Intención emprendedora en estudiantes universitarios: integración de factores cognitivos y socio-personales. Revista Colombiana de Ciencias Sociales, 6(2), 320-340. https://doi.org/10.21501/22161201.1528

(10)Fayolle, A., \& Gailly, B. (2008). From craft to science: Teaching models and learning processes in entrepreneurship education. Journal of European Industrial Training, 32(7), 569593. https://doi.org/10.1108/03090590810899838

(11) Fiduciaria Colombiana de Comercio Exterior [FIDUCOLDEX]. (2017). Programa de Transformación Productiva. https://www.fiducoldex.com.co/sites/default/files/invitacionesarchivos-pdf/TRD\%20PRODUCTIVIDAD\%2014-09-2017.pdf

(12) Galleguillos-Cortés, C., Escobar-Burgos, B. y Hurtado-Cailly, R. (2019). Predictores de la Intención Emprendedora en Alumnos de Pregrado: Un Modelo Basado en Ecuaciones Estructurales. Formación Universitaria, 12(1), 83-94. http://dx.doi.org/10.4067/S071850062019000100083

(13) Gibb, A. (1988). Stimulating new business development (what else besides EDP?). ILO.

(14) Gómez, M. E. (2019). Emprendimientos de base tecnológica: un reto por cumplir. TEC Empresarial, 13(2), 33-44. https://dx.doi.org/10.18845/te.v13i2.4493 
(15) Gutama, M. G. y Jiménez, P. (2019). El emprendimiento y su evolución como una alternativa laboral en el contexto latinoamericano: una revisión de la literatura [Trabajo de pregrado]. Universidad de Cuenca. https://dspace.ucuenca.edu.ec/bitstream/123456789/31772/1/EL\%20EMPRENDIMIENTO \%20Y\%20SU\%20EVOLUCI\%C3\%93N\%20COMO\%20UNA\%20ALTERNATIVA\%20L ABORA.pdf

(16) Hannon, P. (2013). Why is the Entrepreneurial University Important? Journal of Innovation Management, 1(2), 10-17.

(17) Hernández, R., Fernández, C. y Baptista, M. (2014). Metodología de la Investigación (6 6 ed.). Mc Graw Hill.

(18) McClelland, D. (1961). The Achieving Society. Princeton.

(19) Moriano, J., Trejo, E. y Palací, F. (2001). El perfil psicosocial del emprendedor: un estudio desde la perspectiva de los valores. Revista de Psicología Social, 16(2), 229-242.

(20) Oficina de Registro y Control Académico [OCARA]. (2017, Semestre B). Universidad de Nariño.

(21) Osorio, F. y Londoño, J. (2015). Intención emprendedora de estudiantes de educación media: extendiendo la teoría de comportamiento planificado mediante el efecto exposición. Cuadernos de Administración, 28(51), 103-131.

(22) Ruiz, C. (2014, febrero 15). Los principales desafíos de los 20 sectores de clase mundial. La República. https://www.larepublica.co/archivo/los-principales-desafios-de-los-20-sectoresde-clase-mundial-2102556

(23) Sánchez, J. C., Lanero, A. y Yurrebaso, A. (2005). Variables determinantes de la intención emprendedora en el contexto universitario. Revista de psicología social aplicada, 15(1-2), 3760.

(24) Sánchez, J. C., Ward, A., Hernández, B. y Flórez, J. L. (2017). Educación Emprendedora: estado del arte. Propósitos y Representaciones, 5(2), 401-473. http://dx.doi.org/10.20511/pyr2017.v5n2.190

(25) Schumpeter, J. A. (1934). The theory of economic development. Harvard University Press.

(26) Shapero, A. T. (1985). The entrepreneurial event. College of Administrative Science, Ohio State University 
(27) Shapero, A., \& Sokol, L. (1982). The Social dimensions of entrepreneurship. Encyclopedia of Entrepreneurship. http://ssrn.com/abstract=1497759

(28) Tarapuez-Chamorro, E., Flórez-Laiseca, A. M. y Giraldo-Correa, M. P. (2019). Intención emprendedora en Colombia: el caso de los estudiantes de Maestría en Administración. Revista Escuela de Administración de Negocios, (86), 93-113. https://doi.org/10.21158/01208160.n86.2019.2292

(29) Thornton, P. H. (1999). The sociology of entrepreneurship. Annual review of sociology, 25, 19-46. https://doi.org/10.1146/annurev.soc.25.1.19

(30) Valencia, A., Montoya, I. y Montoya, A. (2016). Intención emprendedora en estudiantes universitarios: Un estudio bibliométrico. Intangible Capital, 12(4), 881-922. http://dx.doi.org/10.3926/ic.730

(31) Varela, R. (2008). Innovación empresarial: Arte y ciencia en la creación de empresas ( $3^{\mathrm{a} e d .) .}$ Pearson Educación de Colombia Ltda.

Cómo citar este artículo: Aguilera-Castro, A., Benavides-Pupiales, L. \& Ibarra-Jaramillo, F. (2021). Intención emprendedora en estudiantes de pregrado de la Universidad de Nariño, Colombia. Tendencias, 22(2), 213-238. https://doi.org/10.22267/rtend.212202.174 